NOTE

\title{
Preparation of Vinyl Monomers Containing Bicyclic Ortho Ester Structure
}

\author{
Takeshi Endo, * Mai CAI-Song, ${ }^{*}$ Makoto OKAWARA, ${ }^{*}$ \\ and W. J. BAILEY** \\ * Research Laboratory of Resources Utilization, Tokyo Institute of Technology, \\ Nagatsuta-cho, Midori-ku, Yokohama 227, Japan. \\ **Department of Chemistry, University of Maryland, \\ College Park, MD 20742, USA.
}

(Received October 7, 1981)

\begin{abstract}
KEY WORDS Acrylate / Bicyclic Ortho Ester / Radical Polymerization /
Crosslinking /
\end{abstract}

In our preceding paper, it was reported ${ }^{1-3}$ that the polymerization of bicyclic ketal lactones and spiro ortho carbonates occurs with essentially zero shrinkage or only a slight expansion. Recently, we reported $^{4-5}$ that bicyclic ortho esters such as 1,4dialkyl-2,6,7-trioxabicyclo[2.2.2]octanes can be polymerized with $\mathrm{BF}_{3} \mathrm{OEt}_{2}$ to undergo expansion on polymerization.

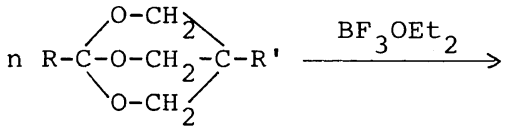

$$
\begin{aligned}
& +\mathrm{CH}_{2} \stackrel{\stackrel{\mathrm{R}}{\mathrm{C}}-\mathrm{l}_{\mathrm{C}}^{\mathrm{C}} \mathrm{CH}_{2} \mathrm{O}-\mathrm{O}-\mathrm{O}}{\mathrm{O}}-\mathrm{n}
\end{aligned}
$$

The present paper describes the preparation and polymerization of acrylates having a bicyclic ortho ester structure resulting from a reaction of the corresponding acryloyl chloride with 1-alkyl-4hydroxymethyl-2,6,7-trioxabicyclo[2.2.2]octanes. These acrylates are considered to be monomers which may function as crosslinking agents without shrinkage.

\section{EXPERIMENTAL}

\section{Materials}

Acryloyl chloride, methacryloyl chloride, pentaerythritol, triethyl orthoacetate and triethyl ortho- propionate were of commercial grade. All solvents used in this study were purified by the usual procedure. 1-Methyl- (I) and 1-ethyl-4-hydroxymethyl2,6,7-trioxabicyclo[2.2.2]octane (II) were prepared by the reaction of triethyl orthoacetate and triethyl orthopropionate with pentaerythritol, respectively, according to the method previously reported by us. ${ }^{5}$

\section{Preparation of Monomers (III-VI) Containing Bicyclic Ortho Ester}

Typical Procedure; Preparation of III

To a solution containing $16 \mathrm{~g}(0.1 \mathrm{~mol})$ of $I$ and $34.6 \mathrm{~cm}^{3}(0.25 \mathrm{~mol})$ of triethylamine in $250 \mathrm{~cm}^{3}$ of dioxane, $14 \mathrm{~g}(0.13 \mathrm{~mol})$ of methacryloyl chloride were added dropwise at $8-10^{\circ} \mathrm{C}$. The reaction mixture was stirred at room temperature for $25 \mathrm{~h}$. After removing the produced $\mathrm{Et}_{3} \mathrm{~N}^{+} \mathrm{HCl}^{-}$salt by filtration, dioxane was evaporated in vacuo and the residue was distilled under reduced pressure in the presence of copper powder $(0.1 \mathrm{~g})$ as an inhibitor to give $17.3 \mathrm{~g}(80 \%)$ of III: bp $103^{\circ} \mathrm{C}(0.25 \mathrm{mmHg})$; IR $1720(\mathrm{C}=\mathrm{O}), 1632(\mathrm{C}=\mathrm{C}), 1150,1047$, and 1027 $\mathrm{cm}^{-1}(\mathrm{C}-\mathrm{O}-\mathrm{C}) ;{ }^{1} \mathrm{H}$ NMR $\left(\mathrm{CDCl}_{3}\right) \delta 1.47(3 \mathrm{H}, \mathrm{s}$, $\left.\mathrm{CH}_{3}\right), 1.95\left(3 \mathrm{H}, \mathrm{s}, \mathrm{CH}_{3}\right), 3.95\left(2 \mathrm{H}, \mathrm{s}, \mathrm{OCH}_{2}\right), 4.02$ $\left(6 \mathrm{H}, \mathrm{s}, 3 \mathrm{OCH}_{2}\right), 5.61-6.09 \mathrm{ppm}\left(2 \mathrm{H}, \mathrm{d},=\mathrm{CH}_{2}\right)$. Anal. Calcd for $\mathrm{C}_{11} \mathrm{H}_{16} \mathrm{O}_{5}: \mathrm{C}, 57.88 \% ; \mathrm{H}, 7.07 \%$. Found: C, $57.76 \%$; H, $7.10 \%$.

IV-VI were also prepared in a similar way.

IV, yield $87 \%$; bp $113^{\circ} \mathrm{C}(0.25 \mathrm{mmHg}) ;{ }^{1} \mathrm{H}$ NMR $\left(\mathrm{CDCl}_{3}\right) \delta 0.94\left(3 \mathrm{H}, \mathrm{t}, \mathrm{CH}_{3}\right), 1.69\left(2 \mathrm{H}, \mathrm{q}, \mathrm{CH}_{2}\right), 1.93$ $\left(3 \mathrm{H}, \mathrm{s}, \mathrm{CH}_{3}\right), 3.90\left(2 \mathrm{H}, \mathrm{s}, \mathrm{OCH}_{2}\right), 3.98(6 \mathrm{H}, \mathrm{s}$, 
$\left.3 \mathrm{OCH}_{2}\right), 5.56-6.04\left(2 \mathrm{H}, \mathrm{d},=\mathrm{CH}_{2}\right)$. IR 1720 $(\mathrm{C}=\mathrm{O}), 1632(\mathrm{C}=\mathrm{C}), 1150$, and $1030 \mathrm{~cm}^{-1}$ (C-O-C).

Anal. Calcd for $\mathrm{C}_{12} \mathrm{H}_{18} \mathrm{O}_{5}: \mathrm{C}, 59.49 \% ; \mathrm{H}, 7.49 \%$. Found: C, $59.42 \% ; \mathrm{H}, 7.52 \%$.

V, yield $73 \%$; bp $92-93^{\circ} \mathrm{C}(0.2 \mathrm{mmHg}) ;{ }^{1} \mathrm{H}$ NMR $\left(\mathrm{CDCl}_{3}\right) \delta 1.45\left(3 \mathrm{H}, \mathrm{s}, \mathrm{CH}_{3}\right), 3.95(2 \mathrm{H}, \mathrm{s}$, $\left.\mathrm{OCH}_{2}\right), 4.01\left(6 \mathrm{H}, \mathrm{s}, 3 \mathrm{OCH}_{2}\right), 5.7-6.6 \mathrm{ppm}(3 \mathrm{H}, \mathrm{m}$, $\left.\mathrm{CH}_{2}=\mathrm{CH}\right)$. IR $1725(\mathrm{C}=\mathrm{O}), 1625(\mathrm{C}=\mathrm{C}), 1126$, and $1020 \mathrm{~cm}^{-1}(\mathrm{C}-\mathrm{O}-\mathrm{C})$.

Anal. Calcd for $\mathrm{C}_{10} \mathrm{H}_{14} \mathrm{O}_{5}: \mathrm{C}, 56.07 \%$; $\mathrm{H}, 6.59 \%$. Found: $\mathrm{C}, 56.03 \%$; H, $6.64 \%$.

VI, yield $75 \%$; bp $103^{\circ} \mathrm{C}(0.6 \mathrm{mmHg}) ;{ }^{1} \mathrm{H}$ NMR $\left(\mathrm{CDCl}_{3}\right) \delta 0.95\left(3 \mathrm{H}, \mathrm{t}, \mathrm{CH}_{3}\right), 1.71\left(2 \mathrm{H}, \mathrm{q}, \mathrm{CH}_{2}\right), 3.95$ $\left(2 \mathrm{H}, \mathrm{s}, \mathrm{OCH}_{2}\right), 4.01\left(6 \mathrm{H}, \mathrm{s}, 3 \mathrm{OCH}_{2}\right), 5.7-6.6 \mathrm{ppm}$ $\left(3 \mathrm{H}, \mathrm{m}, \mathrm{CH}_{2}=\mathrm{CH}\right)$. IR $1725(\mathrm{C}=\mathrm{O}), 1630(\mathrm{C}=\mathrm{C})$, 1133 , and $1030 \mathrm{~cm}^{-1}(\mathrm{C}-\mathrm{O}-\mathrm{C})$.

Anal. Calcd for $\mathrm{C}_{11} \mathrm{H}_{16} \mathrm{O}_{5}$ : C, $57.88 \%$; $\mathrm{H}, 7.07 \%$. Found: $\mathrm{C}, 57.81 \% ; \mathrm{H}, 7.10 \%$.

\section{Polymerization of III-VI}

Polymerization was carried out in sealed tubes. III-IV were polymerized at $70^{\circ} \mathrm{C}$ in $N, N$-dimethylformamide (DMF) using 2,2'-azobisisobutyronitrile (AIBN) $(1.0 \mathrm{~mol} \%$ for monomer $)$ as an initiator. After $15 \mathrm{~h}$, the soluble polymers were purified by dissolution in methylene chloride and this was followed by precipitation in tetrahydrofuran
(THF)- $n$-hexane. An approximate estimation of the molecular weights of the polymers from III, IV, V, and VI based on polystyrene by GPC was made, as shown in Table I.

\section{Copolymerization of III-VI with Vinyl Monomers}

Copolymerization was carried out in a manner similar to that for homopolymerization. The copolymer composition (Table I) was estimated by elemental analysis and NMR $\left(\mathrm{CDCl}_{3}, 60^{\circ} \mathrm{C}\right)$. The composition of III-acrylonitrile (AN) (C, 54.97\%; $\mathrm{H}, \quad 7.06 \% ; \mathrm{N}, 5.33 \%$ ) and III-chloromethylstyrene (CMS) (C, 64.89\%; H, 6.47\%; Cl, 12.63\%) copolymer was determined by elemental analysis. The composition of (III-VI)-styrene (St), (IIIIV)-vinyl acetate (VAc), (III-IV)-methyl methacrylate (MMA) copolymers was also estimated by the NMR proton ratio;

$$
\begin{aligned}
& (\text { III-VI })-\mathrm{St} ; \\
& \quad \mathrm{C}_{2} \mathrm{O}(4.01 \mathrm{ppm}) / \mathrm{C}_{6} \mathrm{H}_{5}(6.2-7.2 \mathrm{ppm}) \\
& (\mathrm{III}-\mathbf{I V})-\mathrm{VAc} ; \\
& \quad \mathrm{C}_{2} \mathrm{O}(4.1 \mathrm{ppm}) /-\mathrm{CH}-(4.7-5.4 \mathrm{ppm}) \\
& (\mathrm{III}-\mathbf{I V})-\mathrm{MMA} ; \\
& \quad \underline{\mathrm{CH}}_{2} \mathrm{O}(4.05 \mathrm{ppm}) / \mathrm{COOCH}_{3}(3.55 \mathrm{ppm})
\end{aligned}
$$

\begin{tabular}{|c|c|c|c|c|c|c|}
\hline \multicolumn{2}{|c|}{ Monomers } & \multirow{2}{*}{$\begin{array}{c}\mathrm{M}_{1} / \mathrm{M}_{2} \\
\text { (mole ratio) }\end{array}$} & \multirow{2}{*}{$\frac{\text { Yield }}{\%}$} & \multicolumn{2}{|c|}{ Copolymer composition } & \multirow{2}{*}{$\mathrm{MW}^{\mathrm{a}}$} \\
\hline $\mathrm{M}_{1}$ & $\mathbf{M}_{2}$ & & & $\mathrm{~m}_{1}$ & $\mathrm{~m}_{2}$ & \\
\hline III & & & 69 & 100 & 0 & $7 \times 10^{4}$ \\
\hline III & St & 0.5 & 58 & 38 & 62 & $2.4 \times 10^{4}$ \\
\hline III & VAc & 0.5 & 40 & 87 & 13 & $5.8 \times 10^{4}$ \\
\hline III & MMA & 0.5 & 65 & 29 & 71 & $11 \times 10^{4}$ \\
\hline III & $\mathrm{AN}$ & 0.5 & 43 & 48 & 52 & $2.4 \times 10^{4}$ \\
\hline III & CMS & 0.5 & 25 & 36 & 64 & $6.5 \times 10^{4}$ \\
\hline III & $\begin{array}{c}\text { St-MMA } \\
(1: 1)\end{array}$ & 0.5 & 45 & 29 & $\begin{array}{l}36 \text { (St) } \\
35 \text { (MMA) }\end{array}$ & $4.9 \times 10^{4}$ \\
\hline IV & & & 48 & 100 & 0 & $10.3 \times 10^{4}$ \\
\hline IV & $\mathrm{St}$ & 0.5 & 53 & 37 & 63 & $4.4 \times 10^{4}$ \\
\hline IV & MMA & 0.5 & 76 & 26 & 74 & $10.9 \times 10^{4}$ \\
\hline IV & $\mathrm{AN}$ & 0.5 & 38 & 48 & 52 & $3.7 \times 10^{4}$ \\
\hline $\mathbf{V}$ & & & 51 & 100 & 0 & $2.5 \times 10^{4}$ \\
\hline $\mathbf{V}$ & St & 0.5 & 34 & 37 & 63 & $2.7 \times 10^{4}$ \\
\hline VI & & & 48 & 100 & 0 & $2.4 \times 10^{4}$ \\
\hline VI & St & 0.5 & 31 & 36 & 64 & $2.6 \times 10^{4}$ \\
\hline
\end{tabular}

Table I. Homopolymerization and copolymerization of III--VI

\footnotetext{
a Based on polystyrene by GPC.
} 
Crosslinking of Copolymers with $\mathrm{BF}_{3} \mathrm{OEt}_{2}$

To a solution containing $1 \mathrm{~g}$ of copolymer in 40 $\mathrm{cm}^{3}$ of methylene chloride, $\mathrm{BF}_{3} \mathrm{OEt}_{2}(3 \mathrm{~mol} \%$ of bicyclic ortho ester moiety) was added at room temperature with stirring. The reaction mixture was stirred at $40^{\circ} \mathrm{C}$ for $8 \mathrm{~h}$ and a solid $(0.95 \mathrm{~g})$ was isolated. The crosslinked polymer was purified by washing it in methanol.

\section{RESULTS AND DISCUSSION}

\section{Preparation of Vinyl Monomers Containing Bicyclic Ortho Ester}

Bicyclic ortho esters, 1-methyl- (I) and 1-ethyl- 4hydroxymethyl-2,6,7-trioxabicyclo[2.2.2]octane (II) were synthesized by the reaction of pentaerythritol with triethyl orthoacetate or triethyl orthopropionate, according to the method reported previously. ${ }^{5}$

$$
\begin{gathered}
\mathrm{R}^{\prime}-\mathrm{C}(\mathrm{OEt})_{3}\left(\mathrm{HOCH}_{2}\right)_{3} \mathrm{CCH}_{2} \mathrm{OH} \\
\longrightarrow \mathrm{R}^{\prime}-\mathrm{CH}_{2}^{\mathrm{O}-\mathrm{CH}_{2}}-\mathrm{CH}_{2}-\mathrm{CH}_{2} \mathrm{OH} \\
\text { I; } \quad \mathrm{R}^{\prime}=\mathrm{Me} \\
\text { II; } \mathrm{R}=\mathrm{Et}
\end{gathered}
$$

By reactions of acid chlorides (acryloyl chloride and methacryloyl chloride) with I or II in the presence of triethylamine, the corresponding monomers (IIIVI) having the bicyclic ortho ester structure were obtained in good yield (see EXPERIMENTAL section).

$$
\begin{aligned}
& \mathrm{CH}_{2}=\prod_{\mathrm{COCl}}^{\mathrm{CR}}+\mathbf{I} \text { or II } \\
& \longrightarrow \mathrm{CH}_{2}=\underset{\mathrm{COOCH}}{\mathrm{C}} \overbrace{\mathrm{CH}_{2}-\mathrm{O}}^{\mathrm{CH}_{2}-\mathrm{CH}_{2}-\mathrm{O}-\mathrm{C}-\mathrm{R}^{\prime}} \\
& \text { III ; } \mathrm{R}=\mathrm{Me}, \mathrm{R}^{\prime}=\mathrm{Me} \\
& \text { IV; } \mathrm{R}=\mathrm{Me}, \mathrm{R}^{\prime}=\mathrm{Et} \\
& \mathrm{V} ; \mathrm{R}=\mathrm{H}, \quad \mathrm{R}^{\prime}=\mathrm{Me} \\
& \text { VI } ; \quad R=H, \quad R^{\prime}=\mathrm{Et}
\end{aligned}
$$

The monomer structure were confirmed by IR, NMR, and elemental analysis, as described in the

\begin{tabular}{|c|c|c|c|c|c|}
\hline \multirow{3}{*}{ Polymer } & \multirow{2}{*}{\multicolumn{2}{|c|}{$\begin{array}{l}\text { Copolymer } \\
\text { composition }\end{array}$}} & \multicolumn{2}{|c|}{$d_{4}{ }^{25} / \mathrm{g} \mathrm{cm}^{-3}$} & \multirow{3}{*}{$\begin{array}{c}\begin{array}{c}\text { Volume } \\
\text { change }\end{array} \\
\frac{\mathrm{a}}{\mathrm{o}}\end{array}$} \\
\hline & & & \multirow{2}{*}{$\begin{array}{l}\text { Soluble } \\
\text { polymer }\end{array}$} & \multirow{2}{*}{$\begin{array}{l}\text { Crosslinked } \\
\text { polymer }\end{array}$} & \\
\hline & $\mathrm{m}_{1}$ & $\mathrm{~m}_{2}$ & & & \\
\hline III & 100 & 0 & 1.278 & 1.282 & -0.3 \\
\hline III-St & 38 & 62 & 1.183 & 1.184 & 0 \\
\hline III-VAc & 87 & 13 & 1.263 & 1.268 & -0.4 \\
\hline III-MMA & 29 & 71 & 1.231 & 1.246 & -1.2 \\
\hline III-AN & 48 & 52 & 1.234 & 1.245 & -0.9 \\
\hline III-CMS & 36 & 64 & 1.248 & 1.248 & 0 \\
\hline III-(St-MMA) & 29 & 36 (St) 35 (MMA) & 1.217 & 1.214 & +0.2 \\
\hline IV & 100 & 0 & 1.239 & 1.252 & -1.0 \\
\hline IV-St & 37 & 63 & 1.215 & 1.189 & +2.2 \\
\hline IV-MMA & 26 & 74 & 1.234 & 1.223 & +0.9 \\
\hline IV-AN & 48 & 52 & 1.247 & 1.242 & +0.4 \\
\hline V-St & 37 & 63 & 1.180 & 1.190 & -0.8 \\
\hline VI-St & 36 & 64 & 1.188 & 1.182 & +0.5 \\
\hline
\end{tabular}
EXPERIMENTAL section.

\section{Polymerization of III-VI}

The radical homopolymerization of III-VI was carried out at $70^{\circ} \mathrm{C}$ in DMF using AIBN to obtain the soluble polymers bearing the bicyclic ortho ester moiety in the side chain as a white powder.

Monomers (III-VI) can also copolymerize with various vinyl monomers such as $\mathrm{St}, \mathrm{MMA}, \mathrm{AN}$,

Table II. Volume change during crosslinking of polymers

a Expansion (+), shrinkage (-). 
VAc, and CMS to yield corresponding copolymers (VII). The results are indicated in Table I.

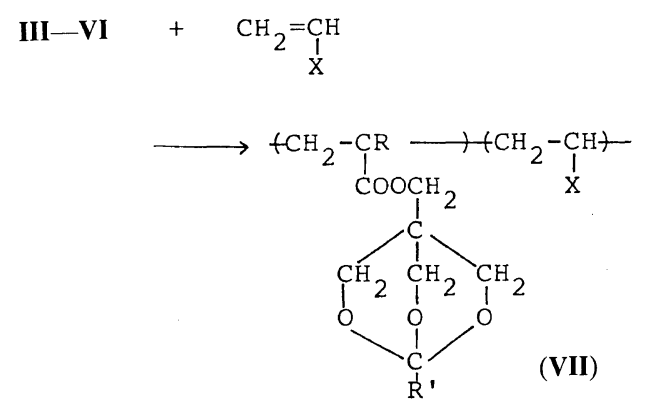

It was found that when the obtained copolymers (VII) were treated with $\mathrm{BF}_{3} \mathrm{OEt}_{2}(3 \mathrm{~mol} \%$ for bicyclic ortho ester) at $40^{\circ} \mathrm{C}$ in methylene chloride, the crosslinked polymers were given probably by the cationic ring-opening transfer reaction of bicyclic ortho ester.

$$
\text { VII } \stackrel{\mathrm{BF}_{3} \mathrm{OEt}_{2}}{\longrightarrow}
$$

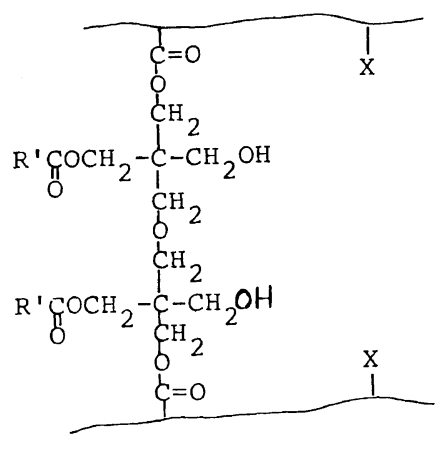

From the measurement of the densities of the soluble copolymers and crosslinked polymers, it was found that these copolymers crosslinked with either no change in volume or only a slight change in volume, as can be seen from Table II. The effects of polymer structure, substituent and content of bicyclic ortho ester on volume change during crosslinking are not entirely clear at the present. It is well known that considerable shrinkage can be observed when polymers crosslink by covalent bond formation. These results are of great interest in consideration of the slight change that takes place in volume.

\section{REFERENCES}

1. T. Endo and W. J. Bailey, J. Polym. Sci., Polym. Chem. Ed., 14, 1745 (1976).

2. T. Endo and W. J. Bailey, Makromol. Chem., 176, 2897 (1975).

3. T. Endo and W. J. Bailey, Makromol. Chem., 177, 3231 (1976).

4. T. Endo, K. Saigo, and W. J. Bailey, J. Polym. Sci., Polym. Lett. Ed., 18, 457 (1980).

5. T. Endo, M. Okawara, and W. J. Bailey, Polym. J., 13, 715 (1981). 